\title{
Free Choice Permission in Defeasible Deontic Logic
}

\author{
Guido GOVERNATORI ${ }^{\mathrm{a}, 1}$ Antonino ROTOLO ${ }^{\mathrm{b}}$

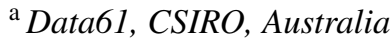 \\ ${ }^{\mathrm{b}}$ Alma Human AI, University of Bologna, Italy
}

\begin{abstract}
Free Choice Permission is one of the challenges for the formalisation of norms. In this paper, we follow a novel approach that accepts Free Choice Permission in a restricted form. The intuition behind the guarded form is strongly aligned with the idea of defeasibility. Accordingly, we investigate how to model the guarded form in Defeasible Deontic Logic extended with disjunctive permissions.
\end{abstract}

Keywords. Free Choice Permission, Disjunctive Permissions, Defeasible Deontic Logic

\section{Introduction}

Free Choice Permission is one of the problems of Deontic Logic where there seems to be a mismatch between the intuition and its formalisation in a deontic language. The problem arises when there is a disjunctive permission, meaning that that one is permitted to chose between two alternatives, let us say $A$ and $B$. The intuition suggests that both alternatives are individually permitted, so $A$ is permitted and $B$ is permitted. Formally, we can represent it by the schema

$$
\mathrm{P}(A \vee B) \rightarrow(\mathrm{P} A \wedge \mathrm{P} B)
$$

The schema is not generally valid in Deontic Logic and, when added as an axiom, it leads to the so-called permission explosion problem [9]: everything is permitted whenever there is a disjunctive permission.

Several logics have been devised to reconcile the intuition with the formal representation for the Free Choice Permission. However, some recent work [7] casts some doubts about the validity of the schema; the following scenario provides a counter-example.

Example 1. When you have dinner with guests, the etiquette allows you to eat or to have a conversation with your fellow guests, but speaking while eating is forbidden.

The example can be formalised as

$$
\begin{aligned}
& \mathrm{P}(\text { eat } \vee \text { speak }) \\
& \text { eat } \rightarrow \mathrm{O} \neg \text { speak } \\
& \text { speak } \rightarrow \mathrm{O} \neg \text { eat }
\end{aligned}
$$

When one performs one of the two alternatives in the disjunctive permission, the other alternative becomes forbidden, and this leads to a contradiction if one assumes (1.1) as an

\footnotetext{
${ }^{1}$ Corresponding author: Guido Governatori, e-mail: guido.governatori@ data61.csiro.au
} 
axiom. This seems to support the view that free choice permission is a pseudo-problem that can be solved by explicitly representing the conjunction when the natural language description supports such a reading [9]. However, the following example [7] indicates that normative reasoning requires the ability to derive an individual permission from a disjunctive permission.

Example 2. A shop has the following policy for clothes bought online. If the size of an item is not a perfect fit, then the customer is entitled to either exchange the item for free, or keep the item and receive a $\$ 10$ refund. However, customers electing to keep the item are not entitled to the refund, and customers opting for the refund are not entitled to exchange the item for free. Furthermore, customers who elect to exchange the item (when entitled to do so) have to return it with the original package.

The representation of this scenario is similar to that of Example 1, namely:

$$
\begin{aligned}
& \neg \text { perfectFit } \rightarrow \mathrm{P}(\text { return } \vee \text { refund }) \\
& \text { return } \rightarrow \mathrm{O} \neg \text { refund } \\
& \text { refund } \rightarrow \text { Oᄀreturn } \\
& \text { Preturn } \wedge \text { return } \rightarrow \text { Ooriginal }
\end{aligned}
$$

When a garment is not a perfect fit, and the customer elects the option to return the item, then, intuitively, the permission of return it holds. However, without Free Choice Permission (or a guarded version of the principle), it is not possible to formally derive the permission, and then we cannot conclude the obligation that it must be returned with the original package.

Based on the discussion so far, it seems that there is the need for a mechanism to derive individual permissions from a disjunctive permission, but the mechanism should be guarded to prevent the derivation of contradictions when some of the alternatives are forbidden. Accordingly, [7] proposed the following guarded version of Free Choice Permission:

From $\mathrm{P}(A \vee B)$ to $\mathrm{P} A$ and $\mathrm{P} B$ provided that it is not the case that $\mathrm{O} \neg A$ or $\mathrm{O} \neg B$.

Furthermore, [7] devised a set of axioms to extend standard classical propositional Deontic Logic to avoid the problem typically caused by Free Choice Permission.

The principle advanced by [7] for a guarded version of Free Choice Permission has an intrinsic defeasible nature. In Defeasible Logic a defeasible conclusion is provable, if there is an argument (rule) in favour, and no arguments for the opposite apply. For Free Choice Permission, the choice among alternatives (disjunctive permission) is the argument in favour of an individual permission (for one of the alternatives) and the inability to derive the prohibition (a prohibition is the opposite of a permission) means that the arguments for the opposite do not hold.

The examples we have provided in this section indicates that there is the need for the formal representation of disjunctive permissions, and that the guarded version of Free Choice Permission is a natural inference pattern of normative reasoning. In the next section, we are going to see how to extend Defeasible Deontic Logic to accommodate such a reasoning pattern. 


\section{Defeasible Deontic Logic}

Defeasible Deontic Logic [5] (DDL) is a sceptical computationally oriented rule-based formalism designed for the representation of norms. The logic extends Defeasible Logic [1] with deontic operators to model obligations and (different types of) permissions, and provides an integration with the logic of violation proposed in [8]. The resulting formalism offers features for the natural and efficient representation of exceptions, constitutive and prescriptive rules, and compensatory norms. The logic is based on a constructive proof theory that allows for full traceability of the conclusions, and flexibility to handle and combine different facets of non-monotonic reasoning. To keep efficiency feasible the language is restricted to literals (atomic propositions and their negation) and deontic literals (literals in the scope of a deontic modality). In what follows, we are going to expand the language to cover disjunctive permissions, and we are going to revise the proof theory to accommodate the extended language. The revised proof conditions are a natural generalisation of the standard proof conditions.

Accordingly, we consider a logic whose language is defined as follows.

Definition 1. Let PROP be a set of propositional atoms, and $\mathrm{O}, \mathrm{P}, \mathrm{P}_{s}$ and $\mathrm{P}_{w}$ the modal (deontic) operators for obligation, permission, strong permission and weak permission respectively. The set Lit $=$ PROP $\cup\{\neg p \mid p \in \mathrm{PROP}\}$ denotes the set of literals. $\sim q$ denotes the complement of a literal $q$ : if $q$ is a positive literal $p$, then $\sim q$ is $\neg p$, and if $q$ is a negative literal $\neg p$, then $\sim q$ is $p$. The set of deontic literals is DLit $=\left\{\square l, \neg \square l \mid l \in\right.$ Lit, $\left.\square \in\left\{\mathrm{O}, \mathrm{P}, \mathrm{P}_{s}, \mathrm{P}_{w}\right\}\right\}$. If $c_{1}, \ldots, c_{n} \in$ Lit, then for $\square \in\left\{\mathrm{P}, \mathrm{P}_{s}, \mathrm{P}_{w}\right\}, \square\left(c_{1} \vee \cdots \vee c_{n}\right)$ is a disjunctive (strong, weak) permission.

We adopt the standard DL definitions of strict rules, defeasible rules, and defeaters [1]. However,

For the sake of simplicity (and space limitations), and to better focus on the nonmonotonic aspects that DDL offers, we only consider defeasible rules; however, the idea presented in the this paper can be easily accommodated in the proof conditions for the full language (see,[5]) to accommodate strict rules and defeaters.

Definition 2. Let Lab be a set of arbitrary labels. Every rule is of the type ' $r: A(r) \hookrightarrow$ $C(r)$ ', where $r \in \mathrm{Lab}$ is the name of the rule; $A(r)=\left\{a_{1}, \ldots, a_{n}\right\}$, the antecedent (or body) of the rule, is the set of the premises of the rule (alternatively, it can be understood as the conjunction of all the elements in it). Each $a_{i}$ is either a literal, a deontic literal, a disjunctive obligation or a disjunctive permission; The set of rules is partitioned in three sets of rules, where the arrow $\hookrightarrow \in\left\{\Rightarrow, \Rightarrow_{\mathrm{O}}, \Rightarrow_{\mathrm{P}}\right\}$ indicates the type of rules: constitutive rule $(\Rightarrow)$, prescriptive rules $\left(\Rightarrow_{0}\right)$ and permissive rules $\left(\Rightarrow_{\mathrm{P}}\right)$. $C(r)$, the consequent (or head) of the rule, is a single literal in case of constitutive rules and prescriptive rules, and a set of literals (intended to be read as a disjunction) in case of permissive rules.

Constitutive rules derive institutional facts, i.e., propositions understood to hold as defined in the underlying normative system. Prescriptive rules are to determine what obligations are in force in the normative system. In contrast, permissive rules generate the permissions in force in the normative system.

The key aspect of the logic is that we consider strong permissions as explicit derogations of obligations to the contrary. Thus, for an explicit permission, we need to have 
a rule that provides the conditions under which something is permitted. We also assume that 'obligation' implies 'strong permission', i.e.,

$$
\mathrm{O} A \rightarrow \mathrm{P}_{S} A
$$

To derive an obligation, we need to have a rule that makes it obligatory. In this view, the strongest way to derogate an obligation is to have a stronger rule that establishes that the same subject matter is forbidden (and we assume the usual interdefinabilty of obligations and prohibitions). For weak permission, we take the view that it is the lack of an obligation to the contrary (or lack of the prohibition). Thus, in case there are no rules for an obligation, then this will enable us to assess that the weak permission holds. The situation where we are not able to derive an obligation because a strong permission derogates it is another case where, in addition to the strong permission, we have the weak permission as well. Every time a strong permission holds, the corresponding weak permission holds a well. Hence, the logic satisfies

$$
\mathrm{P}_{s} A \rightarrow \mathrm{P}_{w} A
$$

Finally, we consider a generic permission, to be used when it is not clear if the permission is either strong or weak, that corresponds to the disjunction of the two, that is:

$$
\mathrm{P} A \equiv \mathrm{P}_{s} A \vee \mathrm{P}_{w} A
$$

Given a set of rules $R$, we use the following abbreviations for specific subsets of rules: $R[q]$ is the subset of $R$ where $q$ is an element of the consequent of the rules in $R$. $R^{c}$ is the subset of the constitutive rules of $R . R^{\mathrm{O}}$ is the subset of the prescriptive rules of $R . R^{\mathrm{P}}$ is the subset of the permissive rules of $R$.

Definition 3. A defeasible theory is a structure $D=(F, R,>)$, where $F$, the set of facts, is a set of literals and modal literals, $R$ is a set of rules and $>$, the superiority relation, is a binary relation over $R$.

A theory corresponds to a normative system, i.e., a set of norms, where for every norm there is a rule modelling it. The superiority relation is used for conflicting rules, i.e., rules whose conclusions are complementary literals, and it just determines the relative strength between the two rules.

Definition 4. A proof $P$ in a defeasible theory $D$ is a linear sequence $P(1) \ldots P(k)$ of tagged literals in the form of $+\partial q,-\partial q,+\partial_{\square} q,-\partial_{\square} q$ for $\square \in\left\{\mathrm{O}, \mathrm{P}, \mathrm{P}_{s}, \mathrm{P}_{w}\right\},+\partial_{\diamond}^{*} q_{1} \vee$ $\cdots \vee q_{m}$ and $-\partial_{\diamond}^{*} q_{1} \vee \cdots \vee q_{m}$ for $\diamond \in\left\{\mathrm{P}, \mathrm{P}_{s}, \mathrm{P}_{w}\right\}$ and $* \in\{f, \max , \min$,$\} , where$ $P(1) \ldots P(k)$ satisfy the proof conditions given in Definitions 6-14.

The tagged literal $+\partial q$ means that $q$ is defeasibly provable as an institutional statement, or in other terms, that $q$ holds in the normative system encoded by the theory. The tagged literal $-\partial q$ means that $q$ the normative system defeasibly refutes $q$. The tagged literal $+\partial_{\circ} q$ means that $q$ is defeasibly provable in $D$ as an obligation, while $-\partial_{\mathrm{O}} q$ means that $q$ is defeasibly refuted as an obligation; similarly for permission, with the difference that for permissions we will consider disjunctions as well. Thus, we can have tagged literals such as $+\partial_{\mathrm{P}_{s}} q_{1} \vee \cdots \vee q_{n}$. The initial part of length $i$ of a proof $P$ is denoted by $P(1 . . i)$.

A rule is applicable for a literal $q$ if $q$ occurs in the head of the rule and all the elements in the antecedent of the rule have already been proved with the appropriate mode. A rule is discarded if at least one of the literals in the antecedent has not been proved. 
Definition 5. Given a derivation $P$, rule $r \in R[q]$ is applicable at step $P(n+1)$ iff for all $a_{i} \in A(r)$, for $\square \in\left\{\mathrm{O}, \mathrm{P}, \mathrm{P}_{s}, \mathrm{P}_{w}\right\}$ and $\diamond \in\left\{\mathrm{P}, \mathrm{P}_{s}, \mathrm{P}_{w}\right\}$ :

1. if $a_{i}=\square l$ then $+\partial_{\square} l \in P(1 . . n)$;

2. if $a_{i}=\neg \square l$ then $-\partial_{\square} l \in P(1 . . n)$;

3. if $a_{i}=\diamond\left(c_{1} \vee \cdots \vee c_{m}\right)$ then $+\partial_{\diamond} c_{1} \vee \cdots \vee c_{m} \in P(1 . . n)$;

4. if $a_{i}=l \in$ Lit then $+\partial l \in P(1 . . n)$.

A rule $r \in R[q, j]$ is discarded iff $\exists a_{i} \in A(r)$ such that

1. if $a_{i}=\square l$ then $-\partial_{\square} l \in P(1 . . n)$;

2. if $a_{i}=\neg \square l$ then $+\partial_{\square} l \in P(1 . . n)$;

3. if $a_{i}=\diamond\left(c_{1} \vee \cdots \vee c_{m}\right)$ then $-\partial_{\diamond} c_{1} \vee \cdots \vee c_{m} \in P(1 . . n)$;

4. if $a_{i}=l \in$ Lit then $-\partial l \in P(1 . . n)$.

The definitions of the negative tags can be obtained from the definitions of the corresponding positive tags by applying the principle of strong negation (that transforms the Boolean operators and quantifiers in their dual, and swapping "applicable" and "discarded" $[2,6]$. For space reasons, we only provide the proof conditions for the positive tags; the exception is the prof condition for $-\partial$ that is given to illustrate the principle of strong negation.

Definition 6. The proof condition to establish when an institutional statement is defeasibly provable is defined as follows:

$+\partial$ : If $P(n+1)=+\partial q$ then

(1) $q \in F$ or

(2.1) $\sim q \notin F$ and

(2.2) $\exists r \in R[q]$ such that $r$ is applicable, and

(2.3) $\forall s \in R[\sim q]$, either

(2.3.1) $s$ is discarded, or either

(2.3.2) $\exists t \in R[q]$ such that $t$ is applicable and $t>s$.

As usual, we use the strong negation to define the proof condition for $-\partial$. Defining the negative proof conditions based on the principle of strong negation ensures that we have a constructive procedure to establish the failure to attempt to satisfy the corresponding positive condition.

Definition 7. The proof condition to establish when an institutional statement is defeasibly refutable is defined as follows:

$-\partial$ : If $P(n+1)=-\partial q$ then

(1) $q \notin F$ and

(2.1) $\sim q \in F$ or

(2.2) $\forall r \in R[q]$ : eit her $r$ is discarded, or

(2.3) $\exists s \in R[\sim q]$, such that

(2.3.1) $s$ is applicable, and

(2.3.2) $\forall t \in R[q]$ either $t$ is discarded or not $t>s$.

The proof conditions for $\pm \partial$ are the standard conditions in defeasible logic, see [1] for the full explanations.

Definition 8. The proof condition to establish when an obligation is defeasibly provable is defined as follows: 
$+\partial_{\mathrm{O}}:$ If $P(n+1)=+\partial_{\mathrm{O}} q$ then

(1) $\exists r \in R^{\mathrm{O}}[p]$ such that $r$ is applicable and

(2) $\forall s \in R[\sim p]$ either

(2.1) $s$ is discarded or

(2.2) $s \in R^{\mathrm{P}}[\sim p]$ and $\exists q \in C(s), q \neq p,-\partial_{\mathrm{O}} \sim q \in P(1 . . n)$ or

(2.3) $\exists t \in R[p]$ such that

(2.3.1) $t$ is applicable and $t>s$ and

(2.3.2) if $t \in R^{\mathrm{P}}[p]$, then $\forall q \in C(t), q \neq p,+\partial_{\mathrm{O}} \sim q \in P(1 . . n)$.

To show that $q$ is defeasibly provable as an obligation we require a prescriptive rule (norm) that is applicable (all the elements of the body of the rule have already been proved with the appropriate mode), Clause (1). Then we have to check that all rules that can generate a conclusion in conflict with the obligation are rebutted (Clause (2)). For an obligation, the rules we have to consider are the prescriptive and permissive rules for $\sim p$. One way to rebut the rule is to establish that the rule is discarded, meaning that at least one of the elements in the body of the rule has been refuted. The other way to rebut an attacking rule is to show that the attacking rule is weaker than an appropriate rule (this step, Clause 2.3, is known as reinstatement). In case we use a permissive rule for reinstatement, we have to ensure that the rule has the potential to derive the permission for the literal we want to prove. Suppose that we have a permissive rule for $a \vee b$, thus the rule would be able to conclude $\mathrm{P}(a \vee b)$, but we do not know which of the two options potentially hold. This means that we cannot use the permissive rule for $a \vee b$ in the reinstatement phase unless we know that all the options but the one in which we are interested are forbidden (Clause 2.3.2): if we want to prove $+\partial_{\mathrm{O}} a$, then $+\partial_{\mathrm{O}} \neg b$ is required, meaning that the prescriptive rule is effectively a rule for $\mathrm{P} a$. As we will see shortly, permissive rules with opposite conclusions are not in conflict with each other. Thus, it might be possible to argue that we cannot use a permissive rule in the reinstatement when the attacking rule is a permissive rule as well. For this variant, we can change the if condition in Clause 2.3.2 with the more restrictive "if $t \in R^{\mathrm{P}}[p]$ and $s \in R^{\mathrm{O}}[\sim p]$ " to obtain the desired result.

We are now ready to provide the proof conditions under which disjunctive permissions (and then individual permissions) can be derived. This requires several steps.

First, we have to determine when a disjunctive permission corresponding to full consequent of a permissive norm is derivable. For this case, given in Definition 9, we have to see that there is an applicable permissive rule, and it is possible to perform at least one of the options without violating any other norm. In other terms, we have to see if we can refute as an obligation at least one of the literals corresponding to one of the permitted alternative. If this is the case, then the disjunctive permission can be assessed as a genuine permission.

Definition 9. The proof condition to establish when a disjunctive permissive norm is defeasibly provable is defined as follows:

If $P(n+1)=+\partial_{\mathrm{P}_{s}}^{f} p_{1} \vee \cdots \vee p_{m}$, then

(1) $\exists r \in R^{\mathrm{P}}\left[p_{1}, \ldots, p_{m}\right]$ such that $r$ is applicable $C(r)=\left\{p_{1}, \ldots, p_{m}\right\}$ and

(2) $\exists p_{i} \in C(r)$ such that $-\partial_{\mathrm{O}} p_{i} \in P(1 . . n)$.

The second case (Definition 10) is to determine what is the largest subset of disjuncts that are not forbidden. While the condition is given in term of a disjunction, the condition can be used to derive a single individual permission (when the permission is the only 
disjunct that is not forbidden). The idea behind this proof condition is similar to the process we described for the derivation of an obligation. The key aspect has two components: First, given an applicable disjunctive permissive rule, all the elements that are not in the disjunction we want to prove are provable as forbidden. Second, only obligation rules can be used to attack the disjunct to be included in the disjunction.

Definition 10. The proof condition to establish when a maximal disjunctive permission is defeasibly provable is defined as follows:

If $P(n+1)=+\partial_{\mathrm{P}_{s}}^{\max } p_{1} \vee \cdots \vee p_{m}$ then

(1) $+\partial_{\mathrm{O}} p_{1}, \cdots+\partial_{\mathrm{O}} p_{m} \in P(1 . . n)$ or

(2) $\exists r \in R^{\mathrm{P}}\left[p_{1}, \ldots, p_{m}\right]$ such that $r$ is applicable and

(2.1) $\forall q \in C(r)-\left\{p_{1}, \ldots, p_{m}\right\},+\partial_{\mathrm{O}} \sim q \in P(1 . . n)$, and

(2.2) $\forall p_{i}, 1 \leq i \leq m, \forall s \in R^{\mathrm{O}}\left[\sim p_{i}\right]$ either

(2.2.1) $s$ is discarded or

(2.2.2) $\exists t \in R\left[p_{i}\right]$ such that $t$ is applicable, $t>s$ and

it $t \in R^{\mathrm{P}}\left[p_{i}\right]$, then $\forall q \in C(t)-\left\{p_{1}, \ldots, p_{m}\right\},+\partial_{\mathrm{O}} \sim q \in P(1 . . n)$.

Consider a theory containing the rules $r_{1}: \Rightarrow_{\mathrm{P}} a \vee b \vee c$ and $r_{2}: \Rightarrow_{\mathrm{O}} \neg a$ where $r_{2}>r_{1}$. In this case, both rules are applicable. However, $r_{2}$ prevails over $r_{1}$ as far as the permission of $a$ is concerned; but there are no rules against $b$ nor $c$. Thus, we can conclude the strong permission of $b \vee c$. To derive an individual (strong) permission, we need that all other options are ruled out as forbidden. Hence, to obtain $\mathrm{P}_{s} b$ we need to have a rule such as $r_{3}: \Rightarrow \bigcirc \neg$ that is stronger than $r_{1}$.

For the third step (Definition 11), consider again the three rules given above. As we have just discussed we have $\mathrm{P}_{s}(a \vee b \vee c)$ given the existence of an explicit rule mandating such permission; we have $\mathrm{P}_{s} b$ from the explicit permissive rule and the prescriptive rules forbidding $a$ and $c$, making $b$ the only really permissive alternative. Is it reasonable to conclude $\mathrm{P}_{s}(a \vee b)$ or $\mathrm{P}_{s}(b \vee c)$ ? These are two permissive disjunctions whose content can be performed (albeit in the same way) without resulting in a violation, thus they appear to be genuine permissions.

Definition 11. The proof conditions to establish when a minimal disjunctive permission is defeasibly provable are defined as follows:

If $P(n+1)=+\partial_{\mathrm{P}_{s}}^{\min } p_{1} \vee \cdots \vee p_{m}$, then

(1) $\exists r \in R^{\mathrm{P}}\left[p_{1}, \ldots, p_{m}\right]$ such that $r$ is applicable and

(2) $\exists p_{j} \in C(r), 1 \leq j \leq n$ such that $+\partial_{\mathrm{P}_{s}}^{\max } p_{1} \vee \cdots \vee p_{j} \in P(1 . . n)$.

The final step is to put the previous three definitions together (Definition 12). A disjunction is provable as a permission if it satisfies one the three previous definitions. In case, one would disallow the last case, it is enough to remove Clause (3) from the definition below.

Definition 12. The proof condition to establish when a disjunctive permission is defeasibly provable is defined as follows:

If $P(n+1)=+\partial_{\mathrm{P}_{s}} p_{1} \vee \cdots \vee p_{m}$, then either

(1) $+\partial_{\mathrm{P}_{s}}^{f} p_{1} \vee \cdots \vee p_{m} \in P(1 . . n)$ or

(2) $+\partial_{\mathrm{P}_{s}}^{\text {max }} p_{1} \vee \cdots \vee p_{m} \in P(1 . . n)$ or

(3) $+\partial_{\mathrm{P}_{s}}^{\text {min }} p_{1} \vee \cdots \vee p_{m} \in P(1 . . n)$ 
For weak permissions we start with the condition to derive individual weak permissions. As we discussed before a weak permission corresponds to the lack of the obligation to the contrary. Hence, we can establish (1) the connection with the failure to derive the opposite obligation and (2)to be able to derive the corresponding strong permission. Then, we can use the condition for an individual permission to lift the condition to the case of a disjunctive weak permission, using the general modal logic inference pattern $\square A \rightarrow \square(A \vee B)$. Notice that differently to the case of $+\partial_{\mathrm{P}_{s}}^{\text {min }}$, the disjunction is not bound to an existing rule and can be used for an arbitrary disjunction. The limitation for strong permissions depends on the nature of permission that requires the explicit existence of a (derogating) permissive rule.

Definition 13. The proof conditions to establish when a weak permission is defeasibly provable and a weak conjunctive permission is defeasibly provable are defined as follows: If $P(n+1)=+\partial_{\mathrm{P}_{w}} p$ then

(1) $-\partial_{\mathrm{O}} \sim p \in P(1 . . n)$ or

(2) $+\partial_{\mathrm{P}_{s}} p \in P(1 . . n)$.

If $P(n+1)=+\partial_{\mathrm{P}_{w}} p_{1} \vee \cdots \vee p_{m}$ then

(1) $\exists p_{i}, 1 \leq i \leq m$ such that $+\partial_{\mathrm{P}_{w}} p_{i} \in P(1 . . n)$.

Finally, the case for a generic permission is just the simple combination of the corresponding conditions for strong and weak permissions and their disjunctions.

Definition 14. The proof conditions to establish when an generic permission is defeasible provable are defined as follows:

If $P(n+1)=+\partial_{\mathrm{P}} p$ then

(1) $+\partial_{\mathrm{P}_{s}} p \in P(1 . . n)$ or

(2) $+\partial_{\mathrm{P}_{w}} p \in P(1 . . n)$.

If $P(n+1)=+\partial_{\mathrm{P}} p_{1} \vee \cdots \vee p_{m}$ then

(1) $+\partial_{\mathrm{P}_{s}} p_{1} \vee \cdots \vee p_{m} \in P(1 . . n)$ or

(2) $+\partial_{\mathrm{P}_{w}} p_{1} \vee \cdots \vee p_{m} \in P(1 . . n)$.

\section{Properties of the Logic}

In this section we are going to present a few results to demonstrate that the behaviour of the logic coincides with the properties identified by [7] for a guarded version of Free Choice Permission.

The first set of results concern some standard properties of Deontic Defeasible Logic, namely consistency and coherence.

Theorem 15. For any Defeasible Theory D,

- for any proof tag \# and any literal or disjunction $l$ it is not possible to have both $D \vdash+\# l$ and $D \vdash-\# l$ (consistency).

- for any literal $l$ it is not possible to have both $D \vdash+\partial_{\mathrm{O}} l$ and $D \vdash+\partial_{\mathrm{O}} \neg l$ (coherence)

These results are immediate corollary of a result published in [6] that essentially specifies that any defeasible logic whose proof tags are defined using the principle of strong negation is consistent and coherent. Notice that the coherence result does not hold for permissions. Indeed it is possible to have permissive rules for opposite conclusions, and 
these are not in conflict with each other: $\mathrm{P} A$ and $\mathrm{P} \neg A$ are not contradictory in Standard Deontic Logic.

The next set of statements offers a description of the relationship among the different deontic modalities and basic properties of the guarded Free Choice Permission approach proposed by [7] and adopted in this work.

Theorem 16. For any Defeasible Theory $D$, and for any literals $l, l_{1}, l_{2}$ and $l_{3}$ :

1. it is not possible to have $D \vdash+\partial_{\mathrm{O}} l$ and $D \vdash+\partial_{\square} \sim l\left(\right.$ for $\left.\square \in\left\{\mathrm{P}, \mathrm{P}_{s}, \mathrm{P}_{w}\right\}\right)$.

2. if $D \vdash+\partial_{\mathrm{O}} l$, then $D \vdash+\partial_{\square} l$ (for $\square \in\left\{\mathrm{P}, \mathrm{P}_{s}, \mathrm{P}_{w}\right\}$ ).

3. if $D \vdash+\partial_{\mathrm{P}_{s}} l$, then $D \vdash+\partial_{\mathrm{P}_{w}} l$.

4. if $D \vdash+\partial_{\mathrm{P}_{s}}^{f} l_{1} \vee l_{2}$ and $D \vdash+\partial_{\mathrm{O}} \sim l_{1}$, then $D \vdash+\partial_{\mathrm{P}_{s}} l_{2}$.

5. if $D \vdash+\partial_{\mathrm{P}_{s}}^{f} l_{1} \vee l_{2} \vee l_{3}$ and $D \vdash+\partial_{\mathrm{O}} \sim l_{1}$, then $D \vdash+\partial_{\mathrm{P}_{s}} l_{2} \vee l_{3}$.

6. if $D \vdash+\partial_{\mathrm{P}_{s}}^{f} l_{1} \vee l_{2}, D \vdash+\partial_{\mathrm{P}_{w}} l_{1}$ and $D \vdash+\partial_{\mathrm{P}_{w}} l_{2}$, then $D \vdash+\partial_{\mathrm{P}_{s}} l_{1}$ and $D \vdash+\partial_{\mathrm{P}_{w}} l_{2}$.

Here we provide the (easy to verify) correspondence with the properties above and the axioms of [7].
1. $\mathrm{O} A \wedge \mathrm{P} \neg A \rightarrow \perp$
2. $\mathrm{O} A \rightarrow\left(\mathrm{P}_{S} A \wedge \mathrm{P}_{w} A\right)$
4. $\mathrm{P}_{s}(A \vee B) \wedge \mathrm{O} \neg A \rightarrow \mathrm{P}_{s} B$
3. $\mathrm{P}_{s} A \rightarrow \mathrm{P}_{w} A$
5. $\mathrm{P}_{s}(A \vee B \vee C) \wedge \mathrm{O} \neg A \rightarrow \mathrm{P}_{s}(B \vee C)$
6. $\mathrm{P}_{s}(A \vee B) \wedge \mathrm{P}_{w} A \wedge \mathrm{P}_{w} B \rightarrow \mathrm{P}_{s} A \wedge \mathrm{P}_{s} B$.

\section{Conclusions}

In this paper, we started with the idea that the guarded version of Free Choice Permission proposed in [7] has essentially a defeasible nature, and we used the idea to create a variant of Defeasible Deontic Logic that accounts for disjunctive permissions and accommodates the guarded Free Choice Permission in a constructive and computationally oriented way. We have shown that the resulting logic satisfies several properties advanced for a logic for the guarded Free Choice Permission. The work on computational complexity and efficient implementation of the logic is left for future work, but, given the structure of the proof conditions (and the similarity with other variants of Defeasible Logic), we expect the complexity to be computationally feasible.

In [4], we have studied how to extend the Defeasible Deontic Logic with conjunctive obligations. The two variants seem to be orthogonal and complement each other. In the current variant, we do not handle negated disjunctions in the scope of permissions. These can be handle by applying De Morgan, and then use the proof theory for conjunctive obligations and permissions. However, the details of such integration are still to be studied.

Another issue we plan to investigate in the future concerns disjunctive obligations. Specifically, we can ask under what conditions it is possible to derive an individual obligation from a disjunctive obligation. Namely, we will examine the so-called Deontic Disjunctive Syllogism

From $\mathrm{O}(A \vee B)$ to $\mathrm{O} B$ provided $\mathrm{\neg} A$.

In Standard Deontic Logic, it can be represented by the following inference rule

$$
\frac{\mathrm{O}(A \vee B) \quad \mathrm{O} \neg A}{\mathrm{O} B}
$$

and it is logically equivalent to axiom $K$ of Standard Deontic Logic

$$
\mathrm{O}(A \rightarrow B) \rightarrow(\mathrm{O} A \rightarrow B)
$$


and to the Deontic Detachment inference rule

$$
\frac{\mathrm{O}(A \rightarrow B) \quad \mathrm{O} A}{\mathrm{O} B}
$$

Deontic Detachment is often related to the so-called Contrary-to-duty paradoxes of Deontic Logic, and it has been debated whether such a principle should be accepted for reasoning with normative systems. While there is debate on Deontic Detachment, the following two examples suggest that the (logically equivalent) Deontic Disjunctive Syllogism is a natural and intuitive patters and as such should be a valid inference pattern.

Example 3. Horty [10, p. 430-431] who proposed the example of two norms "fight in the army or perform alternative service" and "don't fight in the army" (possibly from two different sources), where he claims that the obligation to perform the alternative service follows, from an intuitive standpoint, from the two (partially) conflicting norms.

Example 4. [3] the rules of sudoku $(9 \times 9)$ prescribe that

1. for every cell, for every row, column or block the cell must contain one digit from 1 to 9 ; and

2. for every row, column or block, if a cell contains a digit, no other cell in the same row, column or block can contain that digit.

The first rule establishes that for each cell there are (nine) permissible alternatives, but if a digit already appears in the row or column or block where a cell appears, then it is forbidden to put the digit in the cell.

The work we presented offers some insights into how to handle conjunctive obligations. Nevertheless, we still have to investigate how to properly integrate and coordinate the interactions between disjunctive obligations and disjunctive permissions.

\section{References}

[1] G. Antoniou, D. Billington, G. Governatori, and M.J. Maher. Representation Results for Defeasible Logic. ACM Transactions on Computational Logic, 2:255-287, 2001.

[2] G. Antoniou, D. Billington, G. Governatori, M.J. Maher, and A. Rock. A Family of Defeasible Reasoning Logics and its Implementation. In W. Horn, editor. ECAI 2000. Proceedings of the 14th European Conference on Artificial Intelligence, pages 459-463. IOS Press, 2000.

[3] G. Governatori. A Short Note on the Chisholm Paradox. In G. Casini, L. Di Caro, G. Governatori, V. Leone, and R. Markovich, editors. Proceedings of the 4th International Workshop on MIning and REasoning with Legal texts. CEUR-WS.org, 2020.

[4] G. Governatori, S. Colombo Tosatto, and A. Rotolo. A Defeasible Deontic Logic for Pragmatic Oddity. In F. Liu, A. Marra, P. Portner, and F. Van De Putte, editors. Deontic Logic and Normative Systems: 15th International Conference (DEON2020/2021). College Publications, 2021.

[5] G. Governatori, F. Olivieri, A. Rotolo, and S. Scannapieco. Computing Strong and Weak Permissions in Defeasible Logic. Journal of Philosophical Logic, 42:799-829, 2013.

[6] G. Governatori, V. Padmanabhan, A. Rotolo, and A. Sattar. A Defeasible Logic for Modelling Policybased Intentions and Motivational Attitudes. Logic Journal of the IGPL, 17:227-265, 2009.

[7] G. Governatori and A. Rotolo. Is Free Choice Permission Admissible in Classical Deontic Logic? In F. Liu, A. Marra, P. Portner, and F. Van De Putte, editors. Deontic Logic and Normative Systems: 15th International Conference (DEON2020/2021). College Publications, 2021.

[8] G. Governatori and A. Rotolo. Logic of Violations: A Gentzen System for Reasoning with Contrary-ToDuty Obligations. Australasian Journal of Logic, 4:193-215, 2006.

[9] S.O. Hansson. The Varieties of Permissions. In D. Gabbay, J. Horty, X. Parent, R. van der Meyden, and L. van der Torre, editors, Handbook of Deontic Logic and Normative Systems. College Publications, 2013.

[10] J. Horty. Deontic Modals: Why Abandon the Classical Semantics. Pacific Philosophical Quarterly, 95:424-460, 2014. 